\title{
Segmental schwannomatosis: characteristics in 12 patients
}

\author{
Abdulqader Alaidarous ${ }^{1 *}$ (D), Beatrice Parfait ${ }^{2,3+}$, Salah Ferkal ${ }^{4 \dagger}$, Joëlle Cohen ${ }^{2}$, Pierre Wolkenstein ${ }^{4}$ and \\ Juliette Mazereeuw-Hautier ${ }^{1}$
}

\begin{abstract}
Background: Segmental schwannomatosis is characterized by multiple schwannomas affecting one-limb or less than 5 contiguous segments of spine. Its characteristics are not well described in the literature. Our objective was to better describe the demographic and clinical characteristics of this condition.
\end{abstract}

Methods: This was a retrospective, bi-center study conducted in two French expert centers for neurofibromatosis and schwannomatosis. The clinical, radiographic, pathological and molecular aspects were extracted from patients' clinical records.

Results: Twelve patients with segmental schwannomatosis were identified. Eight were female and 4 were male. The median age at initial symptom was 29 years (range: 6-60 years) and the median age at diagnosis was 34.5 years (range: 13-65 years). Pain was the initial symptom for the majority of patients (7 of 12). The number of tumors was variable with six patients having more than 10 tumors. Peripheral distribution was seen in all patients. Quality of life could be impaired (median Dermatology Life Quality Index score was 4.5 (range: 2-13). The median duration of follow up was 3 years (range: 1-26). Chronic pain was the main complication (9 of 12 patients). Surgical intervention to control chronic pain was performed for 9 patients of whom 5 experienced recurrence of tumors. Molecular investigations revealed heterozygous LZTR1 variants in 3 of 9 patients.

Conclusion: Segmental schwannomatosis is a rare condition that may start early in life and often remains undiagnosed for many years. Pain is the main symptom and consequently could impair the quality of life. Surgery seems to be effective, but recurrences are frequent. Some patients carried heterozygous LZTR1 variants. Further studies are needed to better understand this rare condition.

Keywords: Segmental schwannomatosis, Neurofibromatosis, LZTR1 mutation

\section{Background}

Schwannomas are rare benign tumors of the peripheral nerve sheaths that can appear sporadically as solitary lesions in the general population. When present in a multiple form, they are associated with neurofibromatosis type 2 (NF2) or schwannomatosis.

NF2 is an autosomal dominant condition caused by mutations in the NF2 gene located on chromosome 22q12.2. Affected individuals inevitably develop schwannomas typically affecting both vestibular nerves. Other

\footnotetext{
* Correspondence: abdulqader.alaidarous@gmail.com

${ }^{\dagger}$ Beatrice Parfait and Salah Ferkal contributed equally to this work.

${ }^{1}$ Service de Dermatologie, Hôpital Larrey, CHU de Toulouse, Toulouse, France

Full list of author information is available at the end of the article
}

cerebral tumors can also occur during the course of the disease.

Whereas the hallmark of NF2 is the presence of bilateral vestibular schwannomas, schwannomatosis is characterized by the presence of multiple non-vestibular non-intradermic schwannomas. However, recent reports suggested that patient with schwannomatosis may also develop unilateral vestibular schwannoma or meningioma $[1,2]$.

A recent study was conducted in UK to describe the epidemiology of these two entities. According to the study, schwannomatosis has less than half the prevalence and birth incidence of NF2. Regional prevalence for schwannomatosis was 1 in 126,315 with a calculated birth incidence of 1 in 68,956 cases (prevalence of 1 in 
50,500 and birth incidence of 1 in 27,956 for NF2). Life expectancy was significantly better in schwannomatosis compared to NF2 (mean age at death of 76.9 and 66.2, respectively) [3].

Diagnostic criteria have been developed to distinguish schwannomatosis from NF2. In 1996, MacCollin et al. reported 14 patients with multiple pathologically defined schwannomas without vestibular localization and developed the first research criteria for schwannomatosis [4]. In 2005, they proposed consensus diagnostic criteria for clinical use that were modified the following year by Baser and colleagues to augment their specificities $[5,6]$. These modified diagnostic criteria suggest that patients with schwannomatosis must not fulfill any of the existing sets of diagnostic criteria for NF2, have no evidence of vestibular schwannoma on high-quality magnetic resonance imaging scan (MRI), no first-degree relative with NF2, and no known constitutional NF2 mutation. A new approach was proposed in 2013 by Plotkin et colleagues who take into consideration the genetic mutation as a diagnostic criterion. In addition, they consider the diagnosis of schwannomatosis in those who had a unilateral vestibular schwannoma and also in patients with intracranial meningioma [7].

Several studies have been performed to identify the molecular aspect of schwannomatosis. In 1996, Jacoby et al. described two pathogenic mechanisms that cause schwannomatosis, including mosaic alteration at the NF2 locus and somatic accumulation of NF2-gene mutations [8]. Subsequent, genetic studies showed that the NF2 locus was excluded as the cause for familial schwannomatosis. Hulsebos et al. reported in 2007 a constitutional SMARCB1 (SWI/SNF related, matrix associated, actin dependent regulator of chromatin, subfamily b, member 1) mutation in a family affected by schwannomatosis [9]. Mutations in SMARCB1 are estimated to occur in approximately 40$50 \%$ of familial cases and in $8-10 \%$ of sporadic cases [10]. In 2008, Sestini et al. reported a patient with SMARCB1 mutations associated with somatic NF2 mutations in schwannomatosis-associated tumors [11]. In 2014, a new gene: LZTR1 (leucine zipper like transcription regulator 1) has been reported to be mutated in $80 \%$ of SMARCB1 mutation-negative schwannomatosis patients with somatic molecular alterations of chromosome 22q including distinct different somatic NF2 mutations as well as the loss of $22 \mathrm{q}$ in multiple tumors of a given patient. Subsequent studies performed with schwannomatosis patients with molecularly uncharacterized schwannomas reported mutation detection rates of $22-30$ and $38 \%$ in sporadic and familial cases respectively [12-15].

All these genes, which are located on chromosome $22 q$, appear to act in regulating cell growth in the nervous system. They encode for proteins that act as tumor suppressor and control cell proliferation. Therefore, mutations of these genes may promote cells growth without control or order to form a tumor. However, current genetic testing does not reveal a mutation in all affected individuals, and there may be additional genes responsible for schwannomatosis yet to be discovered.

Segmental schwannomatosis (SS) is a limited form of schwannomatosis, defined by MacCollin as multiple schwannomas located on one limb or on five or less contiguous segments of spine [5]. The pathophysiology is unknown, and the characteristics of SS are not well described in the literature. We report here a series of SS in order to better describe their characteristics, including molecular aspects.

\section{Methods}

\section{Patients}

This was designed as a retrospective, bi-center study. The clinical records of all patients seen between June 2006 and June 2017 in two French expert centers for neurofibromatosis and schwannomatosis (Henri-Mondor Hospital in Paris and Larrey Hospital in Toulouse) were reviewed. All patients with a diagnosis of SS were included in the study. The definition of SS was based on the criteria by MacCollin and the modified diagnostic criteria of Baser [5, 6]. Histological analysis was obtained for all patients either after performing a biopsy as a diagnostic procedure or after a surgical intervention for painful lesions. Patients with incomplete data necessary for confirming the diagnosis (no histological analysis, no cerebral MRI and no MRI of the affected area) were excluded. Data were extracted from patients' clinical records (age of onset, initial presenting symptoms, location of tumors, pain evaluation, ophthalmologic evaluation, quality of life (QOL), genetic data and family history).

Pain was evaluated by using the EVA scale (Pain Comparison Whit Visual Analog Scale: a subjective measure of patient's pain intensity represented by a point between " $0=$ No pain at all" and " $10=$ worst pain imaginable"). QOL was assessed by using the Dermatology Life Quality Index (DLQI: a ten-question questionnaire used to measure the impact of skin disease on the quality of life of an affected person).

\section{Molecular investigations}

After information on genetic testing, signed consent was obtained for 9 of the 12 patients. DNA was isolated from peripheral blood leucocytes using the Maxwell ${ }^{\circ} 16$ system and Maxwell ${ }^{\circ} 16$ LEV Blood DNA Kit (Promega). NF2, SMARCB1 and LZTR1 genes were investigated on the next-generation sequencing (NGS) facility of Cochin Hospital in Paris, France as already reported by Louvrier et al. [16] Briefly, a custom Ampliseq panel targeting the coding sequences of these genes was designed using the 
Ampliseq Designer plugin [17]. Preparation of NGS libraries, amplification, purification, emulsion PCR, enrichment, loading on Ion $316^{\mathrm{TM}}$ chips, sequencing with an Ion Personal Genome Machine ${ }^{\curvearrowleft}\left(\mathrm{PGM}^{\mathrm{TM}}\right)$ System (Thermo Fisher Scientific). Sequence alignment was performed with the Torrent Mapping Alignment Program (TMAP) [18]. Single nucleotide variants (SNVs) and short insertions and/or deletions detection from the bam files was performed using the Torrent Suite Variant Caller (TSVC) plugin from the Torrent Suite Software v5.0.4 [19]. Major calling parameters were chosen as follows to avoid any false negative result: minimum sequencing depth $\geq 5 \mathrm{X}$ for single nucleotides variants (SNVs), multiple nucleotide variants or complex variants and $\geq 10 \mathrm{X}$ for short insertions and/or deletions, minimum allele frequency $(\mathrm{MAF}) \geq 1 \%$ for all using the TSVC. The 3'UTR region of SMARCB1 was investigated by Sanger sequencing.

Copy number variations (CNVs) analysis was also performed by using quantitative values (number of reads for each amplicon of each sample) of the Coverage Analysis plugin on the Ion Torrent Browser 5.0.4.0 (Life Technologies) [16].

\section{Results}

A total of 12 patients with SS were included. Their demographic and clinical characteristics are described in Table 1 . These patients represented $28.6 \%$ of all patients diagnosed with schwannomatosis in the study period of more than 11 years (total of 48 patients: 28 with generalized form, 12 with segmental form and 2 with solitary lesion). Six patients were excluded due to incomplete data.

The majority of patients were female (8 patients: 67\%). The median age at the initial symptom was 29 years (range: 6-60 years) and the median age at diagnosis was 34.5 years (range: 13-65 years). The median duration from the initial symptom to the diagnosis of SS was 3 years (range: 5 months-32 years). A family history of confirmed vestibular schwannoma was identified in one patient (grandfather of patient 11, no available details). Two other patients had grandparents with suspected but not proven neurofibromatosis type 1 . One patient had a father who died from cerebral tumor of unknown origin.

The initial symptom was pain for 7 (58.3\%) patients. For the remaining patients, four (33.3\%) presented with numbness or electrical discharge sensation along the trajectory of the affected nerve and one $(8.3 \%)$ patient presented with painless masses. Other clinical findings included café-au-lait macules in $3(25 \%)$ patients (2 to 6 macules). Patients were otherwise healthy.

Half of the patients had multiple (more than 10) tumors. The remaining patients had 2 to 9 tumors.

SS had a peripheral distribution in all patients (lower limbs location in 7 patients).
A clinical ophthalmologic evaluation was performed for all patients to exclude any stigmata of NF2. All patients had normal evaluation.

One patient was lost to follow up and for the remaining 11 patients, the median duration of follow up was 3 years (range: $1-26$ years).

SS was complicated by chronic pain in 9 (75\%) patients. Seven patients presented initially with pain while electrical discharge was the initial symptom for the other two patients. This pain was either localized to the tumor, radiating along the nerve of origin or felt in areas where there are no adjacent tumors. The pain became intense gradually over time and ranges in intensity from mild to severe. An evaluation of the pain according to EVA scale was available for 7 of the 12 patients. The median score was 8 (range 4-9/10) before any medical or surgical intervention.

Quality of life was assessed in 6 patients using the Dermatology Life Quality Index. The median score was 4.5 (range: 2-13). Only one patient could be considered having an impaired QOL since the score was more than 10. Impairment was related to difficulties in daily activity ranging from mild to moderate disability especially if activity depends on the utility of the affected region. For example, 3 patients with affected hand reported a difficulty during working, writing, sports and manual gesture. This difficulty has been adapted with time and patients became more accommodated to their disease. None reported feeling depressed because of their disease at the time of the study and none used antidepressants. None took day-off from work because of incapability.

Surgical intervention of schwannomas was performed in $9(75 \%)$ patients to alleviate their chronic pain. Recurrence occurred in 5 patients, who needed repeated surgery. Efficacy of the surgery, in term of pain management, was evaluated using EVA scale for 5 patients. The pain disappeared in 3 patients (score equal to zero) and decreased to a low score of 1 or $2 / 10$ for the remaining 2 patients. Medications were introduced in 4 patients: 2 patients (patients 3 and 11) had been treated before the surgical intervention without any notable improvement, 1 patient (patient 12) refused the surgery and in 1 patient (patient 10), the surgery was inapplicable. The main analgesics used were paracetamol, opioid drugs or neuropathic agents such as gabapentin, pregabaline and duloxetine. The efficacy of oral medication was only assessed in 2 patients, pain was reduced but the score remained at 5/10.

Molecular analysis (NF2, SMARCB1 and LZTR1) was performed for 9 (75\%) of the 12 patients. We did not identify any sequence variation in the coding sequences and exon/intron boundaries of NF2 and SMARCB1 genes with an allele frequency threshold of $1 \%$ for SNV and $20 \%$ for CNV. SMARCB1 3'UTR variants (including 


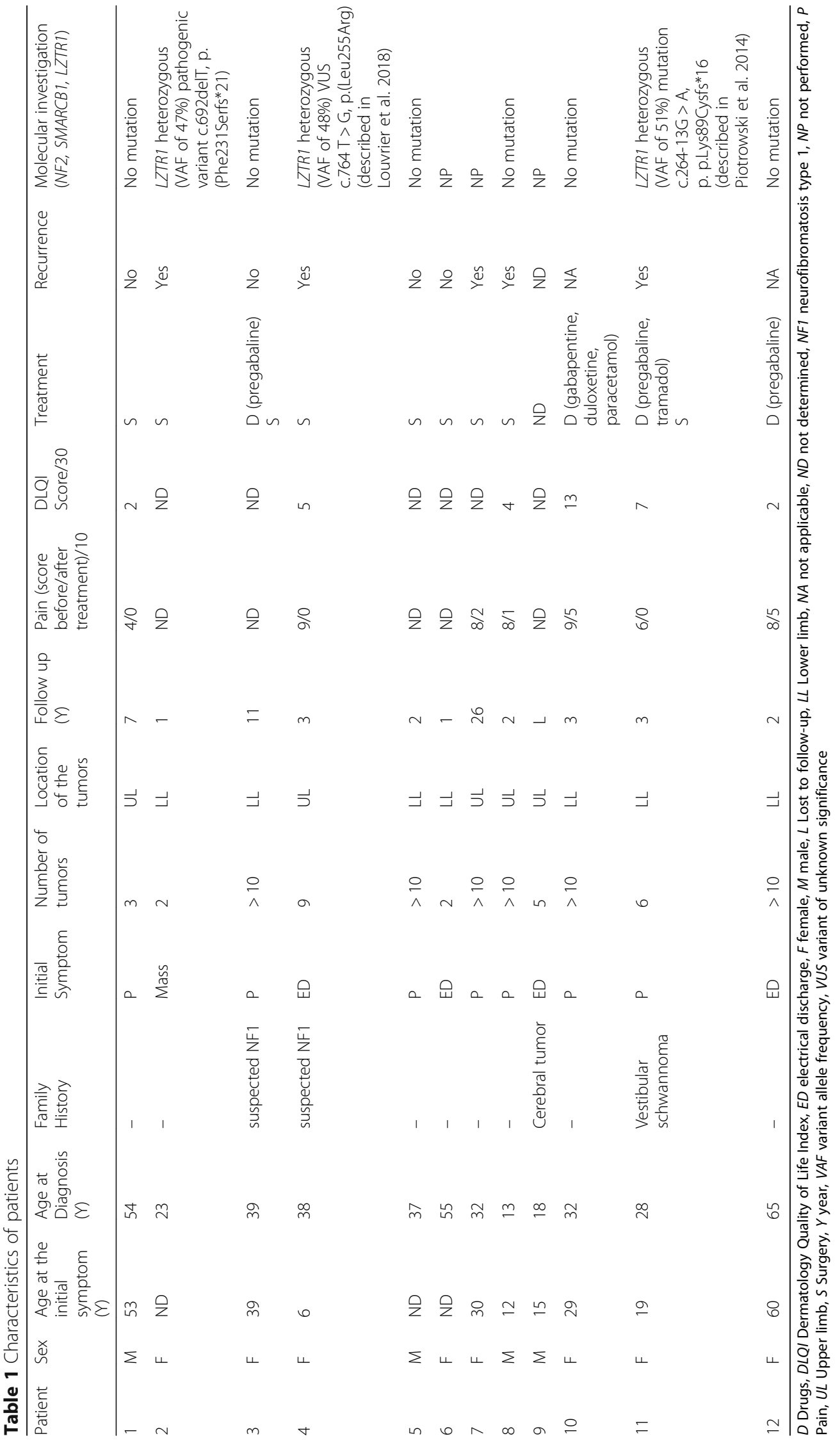


the recurrent c.* $82 \mathrm{C}>\mathrm{T}$ pathogenic variant) were also excluded by Sanger sequencing. LZTR1 heterozygous variants were found in three patients. Patient 2 harbored the c.692delT pathogenic variant in exon 8 corresponding to the predicted deleterious p.(Phe231Serfs*21). Patient 4 harbored c.764 T $>\mathrm{G}$ variant in exon 8 corresponding to the predicted missense p.(Leu255Arg). This patient's variant was already reported [16]. This variant was not described in population databases and was predicted deleterious by several prediction softwares leading to a variant of unknown significance classification according to the American College of Medical Genetics recommendations [20]. Patient 11 carried the heterozygous pathogenic transition c.264-13G > A corresponding to a splicing abnormality c.Lys89Cysfs*29 [12]. These 3 patients had multiple lesions localized either on upper or lower limb.

\section{Discussion}

We report here a series of SS. This series shows that SS is a rare condition (12 cases over a period of 11 years in 2 expert academic centers) that may start early in life and often remains undiagnosed for many years. The number of lesions is variable, and SS often has a peripheral distribution. Pain is the main symptom and QOL could be impaired. Three patients carried LZTR1 mutations.

There are some limitations to our study, related to its retrospective nature. Some patients were excluded for missing data, the duration of follow-up was limited, and one patient was lost to follow-up. Therefore, the possibility of evolution towards generalized schwannomatosis or even NF2 cannot be excluded for all patients. Another limitation is the absence of whole-body MRI scanning that could have discovered asymptomatic schwannomas out of the segmental area. Finally, molecular analysis was performed for only 9 of the 12 patients and due to the unavailability of two distinct tumor specimens, we could not perform molecular analysis to exclude the presence of mosaicism in tissues.

Our review of the literature revealed only 2 small series of 5 and 6 patients with SS from the same Chinese center in 2013. These series described the clinical, histological and radiological aspects but not the molecular features [21, 22]. (Table 2).

In a retrospective analysis of 87 patients with schwannomatosis, 26 had a segmental form. The only reported characteristic was the location: involvement of the leg (35\%), arm (23\%), spine (23\%) or other locations (19\%) [23]. We also found few case reports describing this clinical entity and two studies reporting the molecular analysis without details about their clinical features [24].

With regards to the frequency of SS, it represented nearly a third of all patients with schwannomas in our study, similarly to the series by Merker [23]. It rose up to $75 \%$ in the study conducted by Chen et al. and was much lower in the other series by Wang et al. (1.4\%) $[21,22]$. Our series showed a female predominance, similarly to these 2 other series. In contrast, no female predominance has been reported for the classic form of schwannomatosis.

In our study, the age at the initial symptom was in accordance with the observation of Chen et al. ( 29 vs. 30.3 years). Similarly, the median age at diagnosis in our series was in accordance with Chen and Wang studies (34.5 vs 34.5 and 38 years, respectively) [21, 22].

The majority of our cases presented with pain $(58.3 \%)$, similarly to the 2 other series (50 and 60\%) [21, 22]. The peripheral distribution of the tumors is a common feature between our series and the 2 series of the literature. Nevertheless, all patients in the other series had only an involvement of the upper extremities, contrarily to our patients.

Surgical resection of tumors seems to be effective on pain control. This outcome is similar to what was observed in the other series. However, some locations were not accessible to surgery and then needed other treatment modalities. The percentage of recurrence in our series was much higher than the 2 other series $(55.6 \%$ vs. $16.7 \%$ or $20 \%$ ). Medical treatment is another option. It was considered by our patients as not effective. It was not evaluated in the other series.

QOL was not previously assessed in the literature. Our series showed that SS may affect QOL. We can hypothesize that pain contributes to this impaired QOL.

The pathogenesis of the SS remains unclear. Somatic mosaicism was suggested as the underlying cause by Leverkus who reported one patient with SS presenting with multiples lesions on the left forearm. Biopsy specimens of two different lesions showed two distinct mutations of the NF2 gene, with concomitant loss of heterozygosity in both tumors thus excluding a NF2 mosaic event [24]. Farschtschi studied 5 patients with SS, using magnetic resonance neurography and mutation analysis of NF2, SMARCB1, and LZTR1. In 4 of the 5 patients, subtle fascicular nerve lesions were detected in clinically unaffected extremities. Two patients exhibited LZTR1 germline mutations. This appears contrary to a simple concept of somatic mosaicism and suggests more complex and heterogeneous mechanisms underlying the phenotype of SS than previously thought [25].

In our series, three patients harbored a heterozygous (possibly germline) LZTR1 variant on peripheral blood confirming that the genetic changes causing clinically defined segmental schwannomatosis include LZTR1 gene alterations. The mutation c.264-13G > A in intron 2 has been previously reported by Piotrowski et colleagues in a patient presenting with spinal schwannomas 


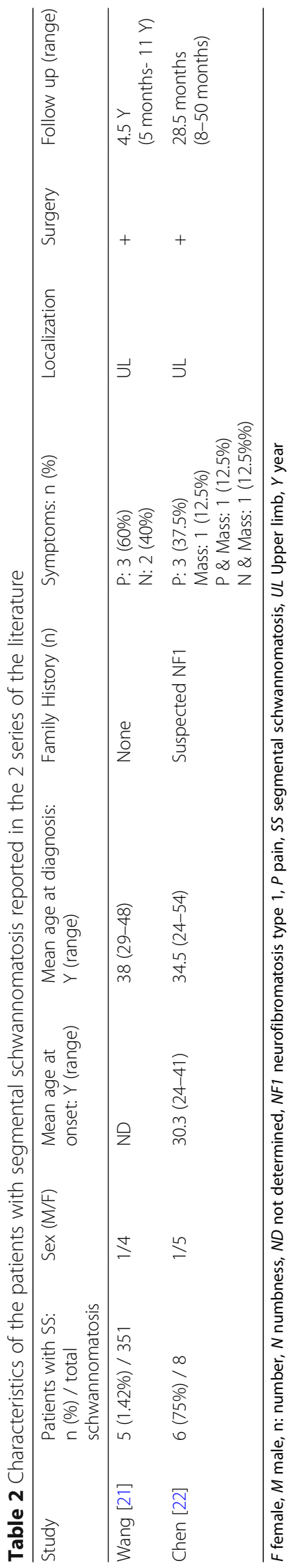


and schwannomas on forearm and abdomen. The patient carrying c.764 $\mathrm{T}>\mathrm{G}$ variant of unknown significance was already reported by Louvrier, and the c.692delT pathogenic variant has not been previously reported in the literature.

One of the main challenges in diagnosing SS is to differentiate it phenotypically from mosaic NF2 or early NF2 since the latter two can fulfill the current criteria of schwannomatosis [26]. Mosaic NF2 can present in the same phenotype as segmental schwannomatosis without vestibular tumor. According to Baser modified criteria, SS is excluded if a constitutional NF2 mutation is found. In this context, we carefully analyzed the NGS data in order to identify mosaic events. We excluded a mosaic event in blood in NF2, SMARCB1 and LZTR1 for 6 out of 9 tested patients with a threshold of $1 \%$ for SNV and $20 \%$ for CNV. This does not exclude the presence of mosaicism in tissues not evaluated in our study or presence at levels below the sensitivity of our method. Further studies on two independent tumors would help to identify the molecular basis for the six LZTR1-negative patients. Together with literature results, our study shows the role of LZTR1 loss of function in the SS phenotype for at least one third of the patients [25].

\section{Conclusions}

In conclusion, SS is a rare and sometimes disabling disease that may start early in life and often remains undiagnosed for many years. Its pathogenesis is still unclear. By describing the characteristics of SS in 12 patients, our series contributes to expand knowledge of disease features. Additional studies are needed to better understand the pathogenesis and help to improve the management of these patients.

\section{Abbreviations}

CNVs: Copy number variations; DLQI: Dermatology Life Quality Index; DNA: Deoxyribonucleic acid; EVA: Pain Comparison Whit Visual Analog Scale; LZTR1: Leucine zipper like transcription regulator 1; MAF: Minimum allele frequency; MRI: Magnetic resonance imaging; NF2: Neurofibromatosis type 2; NGS: Next-generation sequencing; PCR: Polymerase chain reaction; PGM: Personal Genome Machine; QOL: Quality of life; SMARCB1: SWI/SNF related, matrix associated, actin dependent regulator of chromatin, subfamily b, member 1; SNVs: Single nucleotide variants; SS: Segmental schwannomatosis; TMAP: Torrent Mapping Alignment Program; TSVC: Torrent Suite Variant Caller

\section{Acknowledgements}

Not applicable.

\section{Authors' contributions}

AA collected, analyzed and interpreted the patients' clinical data and was the major contributor in writing the article. PB analyzed and interpreted the patient data regarding the molecular aspect and was contributing in writing the articles concerning the molecular aspects. FS made a substantial contribution to the acquisition of the clinical and molecular data. CJ performed the molecular analysis of the specimens and contributed to the data analysis. WP made a substantial contribution to the conception and interpretation of data, writing of the paper. MJ has supervised the work and contributed to all steps, including writing of the article. All authors read and approved the final manuscript.

Funding

Not applicable.

\section{Availability of data and materials}

All data generated or analyzed during this study are included in this published article and its supplementary information files.

\section{Ethics approval and consent to participate}

The project was carried out in accordance with the French legislation of reference framing retrospective non-interventional studies (MR-003, Loi Jardé)

\section{Consent for publication}

Information was given to patients to enable them to oppose the use of their data.

\section{Competing interests}

The authors declare that they have no competing interests.

\section{Author details}

${ }^{1}$ Service de Dermatologie, Hôpital Larrey, CHU de Toulouse, Toulouse, France. ${ }^{2}$ Service de Génétique et Biologie Moléculaires, Hôpital Cochin, Assistance Publique-Hôpitaux de Paris, Paris, France. ${ }^{3}$ UMR INSERM 1016 Institut Cochin \& Université Paris Descartes, Sorbonne Paris Cité, Paris, France. ${ }^{4}$ Service de Dermatologie, Hôpital Henri Mondor, Assistance Publique-Hôpitaux de Paris, Creteil, France.

Received: 21 March 2019 Accepted: 13 August 2019

Published online: 22 August 2019

\section{References}

1. Smith MJ, Kulkarni A, Rustad C, Bowers NL, Wallace AJ, Holder SE, et al. Vestibular schwannomas occur in schwannomatosis and should not be considered an exclusion criterion for clinical diagnosis. Am J Med Genet A. 2012;158A:215-9.

2. Bacci C, Sestini R, Provenzano A, Paganini I, Mancini I, Porfirio B, et al. Schwannomatosis associated with multiple meningiomas due to a familial SMARCB1 mutation. Neurogenetics. 2010;11:73-80.

3. Evans DG, Bowers NL, Tobi S, Hartley C, Wallace AJ, King AT, et al. Schwannomatosis: a genetic and epidemiological study. J Neurol Neurosurg Psychiatry. 2018;89:1215-9.

4. MacCollin M, Woodfin W, Kronn D, Short MP. Schwannomatosis: a clinical and pathologic study. Neurology. 1996;46:1072-9.

5. MacCollin M, Chiocca EA, Evans DG, Friedman JM, Horvitz R, Jaramillo D, et al. Diagnostic criteria for schwannomatosis. Neurology. 2005;64:1838-45.

6. Baser ME, Friedman JM, Evans DG. Increasing the specificity of diagnostic criteria for schwannomatosis. Neurology. 2006;66:730-2.

7. Scott R, Plotkin D, Evans G, et al. Update from the 2011 international Schwannomatosis workshop: from genetics to diagnostic criteria. Am J Med Genet A. 2013:0:405-16.

8. Jacoby LB, Jones D, Davis K, Kronne D, Short MP, Gusella J, et al. Molecular analysis of the NF2 tumor-suppressor gene in schwannomatosis. Am J Hum Genet. 1997;61:1293-302.

9. Hulsebos TJ, Plomp AS, Wolterman RA, Robanus-Maandag EC, Baas F, Wesseling P. Germline mutation of INI1/SMARCB1 in familial schwannomatosis. Am J Hum Genet. 2007;80:805-10.

10. Boyd C, Smith MJ, Kluwe L, Balogh A, Maccollin M, Plotkin SR. Alterations in the SMARCB1 (INI1) tumor suppressor gene in familial schwannomatosis. Clin Genet. 2008;74:358-66.

11. Sestini R, Bacci C, Provenzano A, Genuardi M, Papi L. Evidence of a four-hit mechanism involving SMARCB1 and NF2 in schwannomatosis-associated schwannomas. Hum Mutat. 2008:29:227-31.

12. Piotrowski A, Xie J, Liu YF, Poplawski AB, Gomes AR, Madanecki P, et al. Germline loss-of-function mutations in LZTR1 predispose to an inherited disorder of multiple schwannomas. Nat Genet. 2014;46:182-7.

13. Hutter S, Piro RM, Reuss DE, Hovestadt V, Sahm F, Farschtschi S, et al. Whole exome sequencing reveals that the majority of schwannomatosis cases remain unexplained after excluding SMARCB1 and LZTR1 germline variants. Acta Neuropathol. 2014;128:449-52. 
14. Paganini I, Chang VY, Capone GL, Vitte J, Benelli M, Barbetti L, et al. Expanding the mutational spectrum of LZTR1 in schwannomatosis. Eur J Hum Genet. 2015;23:963-8.

15. Smith MJ, Isidor B, Beetz C, Williams SG, Bhaskar SS, Richer W, et al. Mutations in LZTR1 add to the complex heterogeneity of schwannomatosis. Neurology. 2015;84:141-7.

16. Louvrier C, Pasmant E, Briand-Suleau A, Cohen J, Nitschké P, Nectoux J, et al. Targeted next-generation sequencing for differential diagnosis of neurofibromatosis type 2, schwannomatosis, and meningiomatosis. NeuroOncology. 2018;20:917-29.

17. Ampliseq Designer plugin, Reference IAD51599_119. https://www.ampliseq. $\mathrm{com} /$, Thermo Fisher Scientific.

18. Torrent Mapping Alignment Program (TMAP), (https:/github.com/iontorrent/TMAP, Ion Torrent for Life Technologies.

19. Torrent Suite Variant Caller (TSVC) plugin from the Torrent Suite Software v5.0.4. (https://ioncommunity.thermofisher.com/community/products/ software/torrent_suite, Life Technologies).

20. Richards S, Aziz N, Bale S, Bick D, Das S, Gastier-Foster J, et al. Standards and guidelines for the interpretation of sequence variants: a joint consensus recommendation of the American College of Medical Genetics and Genomics and the Association for Molecular Pathology. Genet Med. 2015; 17:405-24.

21. Wang ZX, Chen SL, Yi CJ, Li C, Rong YB, Tian GL. Segmental schwannomatosis in upper-extremity: 5 cases report and literature review. Beijing Da Xue Xue Bao. 2013;45:698-703.

22. Chen SL, Liu C, Liu B, Yi CJ, Wang ZX, Rong YB, et al. Schwannomatosis: a new member of neurofibromatosis family. Chin Med J. 2013;126:2656-60.

23. Merker VL, Esparza S, Smith MJ, Stemmer-Rachamimov A, Plotkin SR. Clinical features of schwannomatosis: a retrospective analysis of 87 patients. Oncologist. 2012;17:1317-22.

24. Leverkus M, Kluwe L, Röll EM, Becker G, Bröcker EB, Mautner VF, et al. Multiple unilateral schwannomas: segmental neurofibromatosis type 2 or schwannomatosis? Br J Dermatol. 2003:148:804-9.

25. Farschtschi S, Mautner VF, Pham M, Nguyen R, Kehrer-Sawatzki H, Hutter S, et al. Multifocal nerve lesions and LZTR1 germline mutations in segmental schwannomatosis. Ann Neurol. 2016;80:625-8.

26. Baser ME, Friedman JM, Joe H, Shenton A, Wallace AJ, Ramsden RT, et al. Empirical development of improved diagnostic criteria for neurofibromatosis 2. Genet Med. 2011:13:576-81.

\section{Publisher's Note}

Springer Nature remains neutral with regard to jurisdictional claims in published maps and institutional affiliations.

Ready to submit your research? Choose BMC and benefit from:

- fast, convenient online submission

- thorough peer review by experienced researchers in your field

- rapid publication on acceptance

- support for research data, including large and complex data types

- gold Open Access which fosters wider collaboration and increased citations

- maximum visibility for your research: over $100 \mathrm{M}$ website views per year

At $\mathrm{BMC}$, research is always in progress.

Learn more biomedcentral.com/submissions 